\title{
Effects of hand massage and acupressure therapy for mechanically ventilated patients
}

\author{
Şebnem Çınar Yücel ${ }^{1}$ \\ İsmet Eşer ${ }^{2}$
}

\begin{abstract}
Purpose: This research is an experimental study (randomized controlled) which is carried out to investigate the effect on anxiety of hand massage and acupressure therapy in patients having mechanical ventilation support.

Method and material: This randomized controlled research was conducted in Intensive care units of Ege University Faculty of Medicine, Department of Chest Diseases and Dr. Suat Seren Chest Diseases and Chest Surgery Training and Research Hospital between the dates 18 June 2007 and 3 May 2008. The research sample was comprised of a total of 70 patients who met the research criteria in the hand massage and acupressure $(n=35)$ and control $(n=35)$ groups. Data were collected by using Patient Information Form, Patient Record Form and Visual Analogue Scale. It was following patients in experimental and control groups during seven days. It was recorded physiological indicators of anxiety, perceived anxiety and dyspnoea of patients in control group. These patients weren't used hand massage and acupressure therapy. The patients in experimental group weren't used hand massage and acupressure therapy in the first and seventh days. It was recorded physiological indicators of anxiety, perceived anxiety and dyspnoea. The other days, it was used hand massage and acupressure therapy. It was recorded physiological indicators of anxiety, perceived anxiety and dyspnoea This research is an experimental study (randomized controlled) which is carried out to investigate the effect on anxiety of hand massage and acupressure therapy in patients having mechanical ventilation support. Research was conducted in Intensive care units of Ege University Faculty of Medicine, Department of Chest Diseases and Dr. Suat Seren Chest Diseases and Chest Surgery Training and Research Hospital between the dates 18 June 2007 and 3 May 2008. The research sample was comprised of a total of 70 patients who met the research criteria in the hand massage and acupressure $(\mathrm{n}=35)$ and control ( $\mathrm{n}=35$ ) groups. Data were collected by using Patient Information Form, Patient Record Form and Visual Analogue Scale. It was following patients in experimental and control groups during seven days. It was recorded physiological indicators of anxiety, perceived anxiety and dyspnoea of patients in control group. These patients weren't used hand massage and acupressure therapy. The patients in experimental group weren't used hand massage and acupressure therapy in the first and seventh days. It was recorded physiological indicators of anxiety, perceived anxiety and dyspnoea. The other days, it was used hand massage and acupressure therapy. Hand massage and acupressure therapy was limited to 18 minutes. It was recorded physiological indicators of anxiety, perceived anxiety and dyspnoea before and after hand massage and acupressure therapy. It was calculated intensive care unit costs and determined duration in intensive care unit after discharge of patients
\end{abstract}

\footnotetext{
${ }^{1}$ Assoc. Prof., Ege University, Faculty of Nursing, Fundamentals of Nursing Department, İzmir, Turkey; sebnem.cinar@ege.edu.tr

${ }^{2}$ Prof., Ege University, Faculty of Nursing, Fundamentals of Nursing Department, İzmir, Turkey; iseser54@yahoo.com
} 
in experimental and control groups. In the data analysis were used chi square, repeated measures analysis of variance, independent samples $t$ test, paired samples $t$ test, LSD (Least-Significant difference) tests.

Results: According to the research findings, it wasn't found significant as clinical which is the statistically significant difference in the physiological indicators of anxiety, perceived anxiety and dyspnoea except from oxygen saturation.

Conclusion: In conclusion, our results suggest that hand massage and acupressure therapy might effectively relieve perceived dyspnoea and anxiety and reduce physiological indicators of anxiety in patients having mechanical ventilation support. The study provides a research-based intervention model for clinicians caring for MVP.

Keywords: Anxiety, hand massage, acupressure therapy, mechanical ventilation, nurse.

\section{Introduction}

Chronic obstructive pulmonary disease (COPD) is characterized by alterations in the airways and lung parenchyma, resulting in increased respiratory workload (Palm \& Decker 2003). Mechanical ventilator is widely used in the treatment of acute respiratory distress resulting from various reasons such as COPD in the intensive care units. Using mechanical ventilation support is an incredibly stressful experience for patients. Patients describe mechanical ventilation as 'the most inhumane treatment ever experienced', and have reported being miserable most of the time they were intubated (Thomas, 2003). Anxiety and dyspnoea are reported as major problems for most patients requiring a ventilator (Chlan 2009, Johnson \& Sexton 1990, Kress 2006, Rotondi et al. 2002, Tsay et al. 2005). Furthermore, high levels of perceived dyspnoea with anxiety are often reported as major factors interfering with weaning trials, and lead patients to long-term respirator dependency (Korhan et al. 2011, Rotondi et al. 2002). Anxiety and dyspnoea cause increased sympathetic nervous system activation, lead to arterial and venous constriction, myocardial stimulation, bronchoconstriction, increased airway resistance, increased laboured breathing, and increased oxygen demand, thus increasing weaning patients from mechanical systems (Johnson \& Sexton 1990).

Few documented interventional studies have addressed the symptoms of anxiety and dyspnoea in patients using mechanical ventilation. Thus, there is a need to develop effective methods to manage problems such as dyspnoea or anxiety in these patients using a ventilator.

\section{A review of the literature}

Sedative and anxiolytic agents are used in order to take the anxiety under control in the patient having mechanical ventilation support. While these agents are expensive, they can also lead to undesired results such as nausea, vomiting, muscle weakness and atrophy, prolongation of the 
Çınar Yücel, Ş., \& Eşer, İ. (2015). Effects of hand massage and acupressure therapy for mechanically ventilated patients. International Journal of Human Sciences, 12(2), 881-896. doi:10.14687/ijhs.v12i2.3054

time when ventilatory support is needed, increase of the infection risk, changes in the mental state, hypotension, increase of the risk of pneumonia associated with ventilator, bradycardia, coma, respiratory depression, renal failure and even death. These consequences necessitate the application of different treatments that will reduce the length and in turn, the cost of ventilation and will provide comfort for the patients on mechanical ventilators by eliminating the anxiety (Sarıcaoğlu et al. 2005). Thus, nursing interventions that will alleviate anxiety and dyspnea perceived in the patients who need ventilatory support are of vital importance.

Massage which is the planned and purposeful action of touching improves the relationship between patient and nurse, reduces the anxiety level, provides comfort and relaxation, reduces the need of analgesics and sedation. Hand massage can be integrated into routine nursing care activities easily and it is a noninvasive intervention that can be learned and applied easily and enables the communication between the nurse and patient thanks to personal care and touching (Fraser \& Ross 1993, Dunn et al. 1995). According to the stress theory of Selye, massage is a holistic physiological response that occurs in hypothalamus which causes a general increase or decrease in stimulating central nervous system. Massage, which provides comfort by decreasing tender in muscles, is the opposite of stress response. Eliminating anxiety or providing comfort can be obtained through the decrease in muscle spasms (Fraser \& Ross 1993). Studies demonstrate that massage could be used in treatments of illnesses such as anxiety, stress and pain through decreasing muscle tension (Fraser \& Ross 1993, Dunn et al. 1995, Ersser et al. 1998). In the study that Kolcaba et al. (2006) examined the effect of hand massage on comfort in elderly patients staying in nursing home, they found that hand massage was significantly effective in first weeks in the experiment group but not significant in the following weeks. Kolcaba et al. (2004) in another study detected that hand massage increased the comfort of nursing home patients. However, no study examining the effect of hand massage on anxiety of the patients having mechanical ventilation support is available in the literature.

Acupressure is a non-invasive therapy that is based on Traditional Chinese Medicine (TMC), and might offer a valuable therapy modality to manage symptoms in these patients. The traditional technique(s) appears to be effective in the treatment of pain, nausea, fatigue, sleep disturbances, drug detoxification and in aiding stroke victims (NIH 1997, Tsay \& Chen 2003, Tsay 2004, Hmwe et al. 2015, Au et al. 2015, Dreyer et al. 2015). Acupuncture stimulates neurotransmitters and adrenocorticotropic hormones, and studies have supported its use as an endorphin-mediated mechanism in treat analgesia (Hsu 1996). Similar to acupuncture (a very mild but invasive procedure), acupressure therapy applies mild, non-invasive pressure to certain meridian points on the body to relieve various symptoms. 
Dyspnoea is a frequent complaint in patients with COPD, especially in those using mechanical ventilation, and is a strong subjective experience of physiological distress. In the study conducted by Tsay et al. (2005) investigated the management of symptoms of dyspnoea in patients COPD who are using a ventilator. They determined a statistically significant healing in dyspnoea, anxiety and physiological indicators of dyspnoea in patients who were applied acupressure with COPD in the support of mechanical ventilation. This clinically insignificant decrease was considered as important by Tsay et al. (2005) and it was supported that the practice of acupressure was effective. Also, three related studies were identified within a community of patients with COPD. In the first study, 12 matched pairs of such patients received acupuncture or placebo acupuncture. Following 3 weeks of treatment, the acupuncture group showed a statistically significant improvement in perceived breathlessness; however, objective measures of lung function remained unchanged in both groups (Jobst $e t$ al. 1986). The second study assessed the effects of self-administered acupressure as an adjunct to a pulmonary rehabilitation programme (PRP) for the relief of symptoms of dyspnoea in patients with COPD. Real acupressure was found to be more effective than sham acupressure in reducing dyspnoea, as measured by a visual analogue scale (VAS) (Maa et al. 1997). However, these reports did not give the locations of the acupoints used. A recent study investigated the effectiveness of acupressure in improving dyspnoea in 44 patients with COPD. The results demonstrated that the true acupoints acupressure group improved statistically significantly in dyspnoea scores compared with the control group (Wu et al. 2004). However, the protocol for acupressure treatment was very complicated and might not be realistic for use by busy clinicians in practice.

Anxiety often accompanies symptoms of perceived dyspnoea. Acupuncture has been reported to reduce anxiety symptoms statistically significantly better than amitriptyline in patients with depression (Yang et al. 1994). Similarly, a randomized controlled trial of 43 patients with anxiety disorders showed statistically significant improvements after 10 acupuncture treatments (Eich et al. 2000). Additionally, a study evaluated the effect of acupuncture on presurgical anxiety, with statistically significant beneficial differences found only in the group receiving acupuncture at the 'relaxation points' of the body (Wang \& Kain 2001).

In conclusion, many patients require mechanical ventilator support, and confront the difficulty of a weaning process as a result. Mechanical ventilation treatment is a very stressful experience, and this should not be ignored by nurses taking care of these patients. Patients using ventilators exhibit high levels of perceived dyspnoea, and the accompanying anxiety interferes with weaning protocols and leads to unwanted dependency on ventilator systems for survival. Preliminary findings from published studies suggest that acupuncture or acupressure might be 
effective in helping patients with COPD with symptoms of dyspnoea and/or anxiety on an outpatient basis. In a study made by Tsay et al. (2005) has been conducted to assist these patients to decrease their perceived dyspnoea and anxiety but the efficiency of acupressure was investigated in their study. A number of these studies are limited by the reliance on small sample sizes and lack of appropriate control groups. As can be seen, there are studies examining the efficiency of acupressure on this patient group in the world, particularly in the Far East. However, acupressure is not a method that is known and preferred to apply in Turkey. As it is thought that the application of an unknown method to this patient group whose anxiety level is high can increase the anxiety level, it has been decided to examine the acupressure together with the efficiency of hand massage that is a method known and applied in Turkey. Given that hand massage and acupressure therapy is low cost, easily mastered and performed and non-invasive, this treatment technique should be investigated using methodologically sound, well- controlled studies.

\section{Purpose}

The aim of this study was to apply an experimental design to examine the effectiveness of massage and acupressure therapy to perceived dyspnoea, anxiety, heart rates (HRs), respiratory rates (RRs), blood pressure and oxygen saturation values in patients with mechanical ventilation system usage.

\section{Method and material}

\section{The place and time of the study}

The study was conducted in two intensive respiratory care units affiliated with two hospitals between the dates 18th June 2007 and 3rd May 2008 in Izmir/ Turkey.

\section{Population and sample selection}

The research sample was comprised of a total of 70 patients who met the research criteria in the hand massage and acupressure $(\mathrm{n}=35)$ and control $(\mathrm{n}=35)$ groups.

\section{In the research scope, patients selected which are;}

- Under support of invasive mechanic ventilation,

- In PS and SIMV modes of mechanic ventilation,

- In intensive care unit for at least 48 hours,

- At the age of 18 and above,

- Not diagnosed with neurologic and psychiatric disease, 
- Not getting sedation therapy at the times when hand massage applied,

- Not using neuromuscular blocker,

- Not under support of high dose inotrop (dopamine and/or dobutamine; not exceeding 10 $\mathrm{mcg} / \mathrm{kg} /$ hour)

- Have $\mathrm{PaO} 2$ under 60 mmHg', PaCO2 above $50 \mathrm{mmHg}$ and $\mathrm{O} 2$ saturation not under 90 in the arterial blood gas analysis

- Do not have hypoglycemia, sepsis table or primary solid organ malignity,

- Have hemodynamic stability,

- Do not received IV infusion at the back of hands,

- Conscious and can communicate,

- Have Glaskow Coma Scale values between 9-15,

- Do not have hearing problems,

- Do not hold any objections for application of hand massage and acupressure (no development of thrombus, ecchymosis, hematoma, phlebitis on hands or arms, intact skin integrity, undeformed hands, nails, or fingers).

Total of 134 patients have been excluded from the research scope for reasons as; weaning from ventilator support before seven days or ventilator mode change $(n=102)$, worsening of general condition $(n=12)$, withdrawal from the study during the research $(n=4)$, starting of IV infusion from the hand during the research $(n=10)$ and development of edema on the hand $(n=6)$.

\section{Power analysis}

To obtain the power of sample size, Power Analysis applied on the first ten patients. According to these; on 0.05 level of significance power values of $\% 94$ for systolic blood pressure $\left(n_{1}=3, n_{2}=3\right), \% 94$ for dialostic blood pressure $\left(n_{1}=12, n_{2}=12\right), \% 96$ for heart rate $\left(n_{1}=4, n_{2}=4\right)$, $\% 80$ for respiration rate $\left(n_{1}=31 n_{2}=31\right), \% 81$ for perceived anxiety $\left(n_{1}=9 n_{2}=9\right)$ and $\% 81$ for perceived dyspnea $\left(n_{1}=26 n_{2}=26\right)$ have been determined.

\section{Type of study}

The study was a two-group experimental design with repeated measures. 


\section{Data collection}

\section{Data collection method}

Qualified patients who consented to participate were randomly assigned to either the experimental or control group. Only the researcher was aware of which treatment the patients were receiving. Their usual caregivers were uninformed as to their treatment group.

It was following patients in experimental and control groups during seven days. It was recorded physiological indicators of anxiety and dyspnoea, perceived anxiety and dyspnoea of patients in control group in the afternoon during seven days. These patients weren't used hand massage and acupressure therapy. The patients in experimental group weren't used hand massage and acupressure therapy in the afternoon in the first and seventh days. It was recorded physiological indicators of anxiety, perceived anxiety and dyspnoea in the first and seventh days. The other days, it was used both hand massage (during ten minutes) and acupressure therapy (during eight minutes). It was recorded physiological indicators of anxiety and dyspnoea, perceived anxiety and dyspnoea before hand massage and acupressure therapy in the afternoon by the researcher.

\section{Data collection tools}

Data were collected by using patient information form, patient record form, Visual Analogue Scale (VAS). Developed by the researcher in patient information form, there are five questions about diagnosis of patient, age, sex, length of stay on mechanical ventilation and in intensive care unit.

\section{Patient Record Form}

In this research we utilized important, certain and objective indicators such as hemodynamic values like heart, respiration rate and blood pressure values as physiological indicators of anxiety or relaxation and oxygen saturation as dyspnoea indicator. On the patient record form were recorded hemodynamic values of patient such as blood pressure, heart and respiration rate and oxygen saturation before the hand massage and acupressure therapy. Mean blood pressure, respiratory, heart rates and oxygen saturation values were obtained at same time via a bedside cardiac monitor in the afternoon. 


\section{Visual Analogue Scale}

Vertical $100 \mathrm{~mm}$ Visual Analogue Scale (VAS) which is reliability and validity for using on these critical patients (Tsay et al. 2005) was used to measure perceived anxiety and dyspnoea everyday before the massage and acupressure. Patients informed on how to use VAS. Patient has been asked if he or she has anxiety and respiration problem and the spot pointed by the patient on Visual Analogue Scale was identified. Two separate VASs were used on identification of perceived anxiety and perceived dyspnoea.

\section{Hand Massage and Acupressure protocol}

In the Turkish community, hand massage is a method which is widely used in order to eliminate anxiety and provide relaxation. Even though acupressure therapy is not a method used and applied in our community, it has been decided to apply it together with hand massage in order to strenghten the effect of hand massage by taking its effects specified in the literature into account and to prevent it to be an unfamiliar method to the patients.

At the result of literature examination it is found that in the studies examining the effect of hand massage on relief and comfort separate massage programmes like 5 and 10 minutes were applied (Kolcaba et al. 2004, 2006). Hand massage for 10 minutes which was used by Kolcaba et al. (2006) was chosen from these programmes. Permission was taken from the researcher for the application of this hand massage program in our study.

It was used an acupressure protocol used by Tsay et al. (2005) based on the literature and in consultation with two licensed Traditional Chinese Medicine physicians. Two acupoints of Neiguan (PC6) and Hegu (LI4) in both hands were selected to decrease symptoms of dyspnoea and anxiety. Neiguan (PC6) is a pericardium meridian, indicated for symptoms of palpitation, dyspnoea and irritability. Hegu (LI4) is a large intestine meridian, with medical implications for treating pain and swelling of neck, face, nose, mouth, throat and respiratory tract congestion. Low pressure applied on every single point for 4 minutes. The precision of acupressure was confirmed if patients felt sore, numb, heavy, or distended as a result of acupressure massage. Each acupressure therapy was limited to 8 minutes of acupoints massage ( 4 minutes per acupoint).

Patients in the experimental group received hand massage and acupressure therapy every day for 6 days at mid-afternoon. Researcher received training on theorical information and practicing to apply hand massage and acupressure legally and safely from a Traditional Chinese Medical Specialist and three Massage Therapists. 
Before applying hand massage and acupressure, patients' hands and arms are examined for thrombus, ecchymosis, hematoma, phlebitis, skin integrity and color to determine whether there is a problem for the massage.

\section{Research ethics}

Approval from the college and respective hospitals' Research and Ethics Committees was obtained before initiation of the study. Potential patients were approached and fully informed of the purpose of the research, its methods, benefits and risks. Patients and their families gave written consent once they had agreed to participate. Anonymity and confidentiality were assured and participants could withdraw from the study at any point without affecting their subsequent treatment.

\section{Evaluation of data}

All statistical analyses were carried out using the Statistical Package for Social Science (SPSS 17.0, Chicago, IL, USA). Characteristics of the participants were summarized by means and standard deviations. The Students't-test and chi-square test were used, as appropriate, to analyse group differences. Value changes of study outcomes (BP, HR, RR, Oxygen saturation, dyspnoea and anxiety) from day 1 to day 7 were expressed in both experimental and control groups. In the data analysis were used Repeated Measures Analysis of Variance and LSD (Least-Significant difference) tests.

\section{Results}

\section{Demographics}

All patients had diagnosis of COPD. The sample consisted of 70 patients -52 men and 18 women - with a mean age of $64.90 \pm 12.056$ years (range 27-87). Patients had been using mechanical ventilation for a mean of $8.31 \pm 6.35$ (range 3-28) days. Their RRs ranged from 18 to 32 breaths per minute while on PS or SIMV mode at baseline. It was determined that the patients in control and experimental groups taken into the scope of the research showed a homogenous distribution in terms of age, gender, length of stay on mechanical ventilation and in intensive care unit until the day of inclusion to the research $(\mathrm{p}>0.05)$. 
Çınar Yücel, Ş., \& Eşer, İ. (2015). Effects of hand massage and acupressure therapy for mechanically ventilated patients. International Journal of Human Sciences, 12(2), 881-896. doi:10.14687/ijhs.v12i2.3054

\section{Changes in HRs and RRs}

All 70 patients completed 7 data points, which included baseline (day 1), during intervention (days 2-6), and follow- up (day 7) periods. After controlling for potential confounding variables, mean HR at baseline was $84.37 \pm 9.63$ for the experimental group and $85.37 \pm 9.34$ for the control group; there were no statistically significant differences between the groups ( $p>0.05)$. The profile of changes in mean HR for the experimental group, HR declined gradually the second day of therapy (day 3) to a low point at day 7. However, for the control group, HR remained basically unchanged throughout the study. HRs in the experimental group changed during intervention and follow-up, compared with the control group (Figure 1).
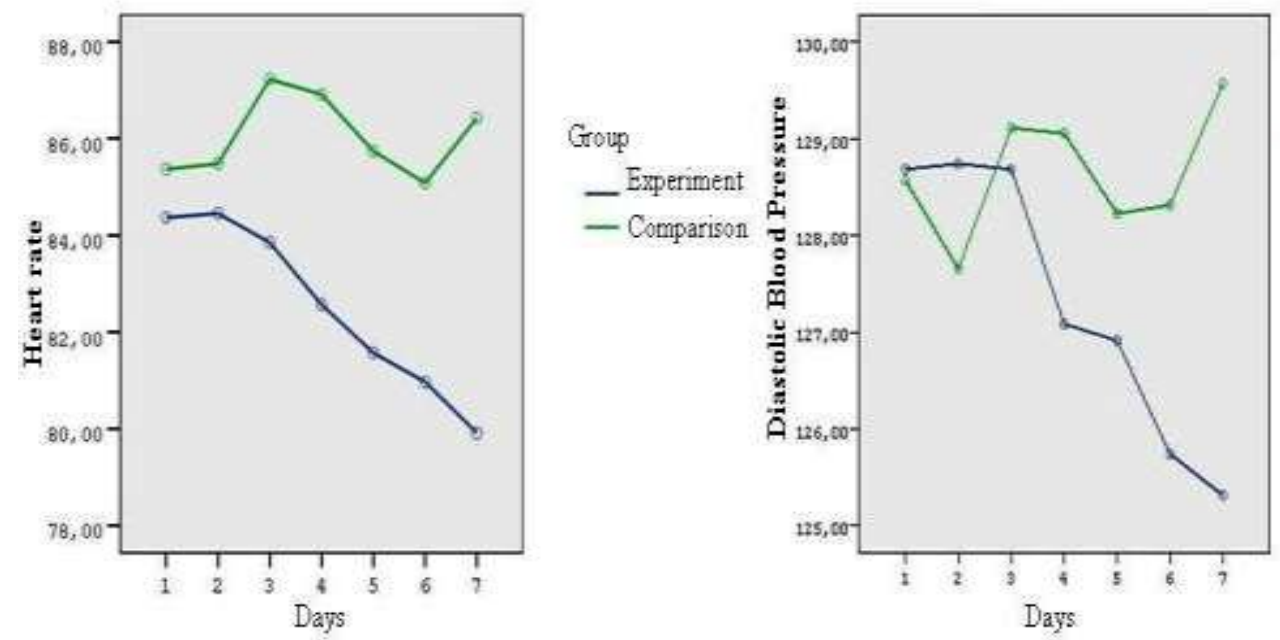

\section{Group \\ -Emeriment}

- Comparison
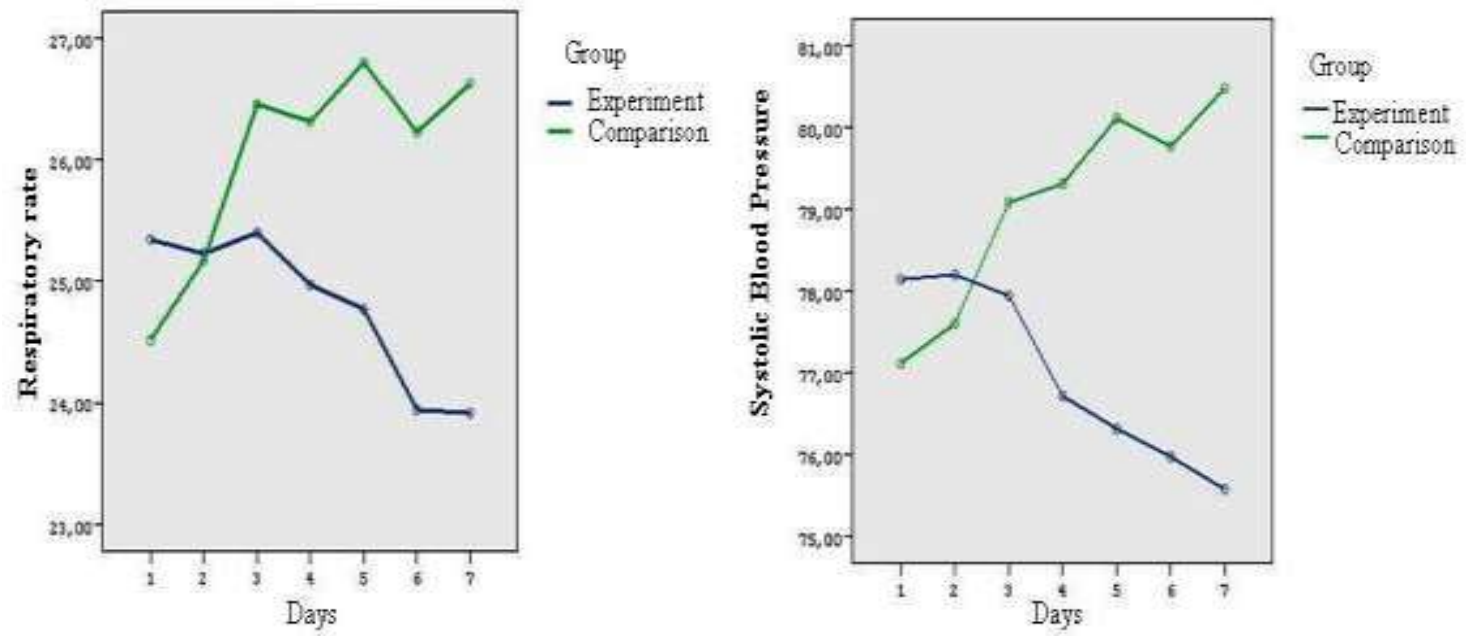

Figure 1. Changes in respiratory, heart rates and blood pressure ralues over time at baseline (day 1), and during massage and acupressure therapy (days 2-6) and follow-up (day 7). The data are shown as mean

The mean RR at baseline was $25.34 \pm 3.62$ for the experimental group and $24.51 \pm 2.70$ for the control group; there were no statistically significant differences between them for $R R(t=1.08$, $\mathrm{p}=0.28$. The profiles of changes in the mean RRs differed between the two groups. For those in the experimental group, RR gradually declined overtime, reaching the lowest value at 7 days . In 
contrast, for those in the control group, RRs increased over time. Patients in the experimental group had lower RR than those in the control group during intervention (days 2-6), and follow- up (day 7$)$ periods $(p>0.05)$ (Figure 1$)$.

\section{Changes in Blood Pressure Values}

The mean systolic and diastolic BP respectively at baseline was $128.69 \pm 10.41,78.14 \pm 5.94$ for the experimental group and $128.57 \pm 13.55,77.11 \pm 6.21$ for the control group. There was no statistically significant difference between groups at baseline ( $p>0.05)$. In the experimental group, systolic and diastolic BP values gradually decreased from day 3 to a lower level on day 7 . Conversely, control group BP values didn't change over time (Figure 1).

\section{Changes in Oxygen Saturation Values}

There were no statistically significant differences between the two groups over time in oxygen saturation value $(\mathrm{p}>0.05)$.

\section{Changes in Perceived Dyspnoea and Anxiety}

Mean perceived dyspnoea for the VAS (0-100) at baseline was $76.42 \pm 7.04$ for the experimental group and 67.02 \pm 8.30 for the control group, and indicating that study participants had high levels of perceived dyspnoea; however, there was a statistically significant difference between the groups $(t=5.10, \mathrm{p}=0.00)$.

The profile of changes in mean perceived dyspnoea differed for experimental and control groups. In the experimental group, perceived dyspnoea gradually declined overtime, reaching the lowest value at day 7. In contrast, in the control group, perceived dyspnoea increased overtime from baseline throughout the follow-up day (Figure 2). The time-dependent changes were also a statistically significant indication that values for the experimental group decreased daily $(p<0.05)$. All these results support the efficiency of hand massage and acupressure therapy in decreasing perceived dyspnoea (Figure 2). 


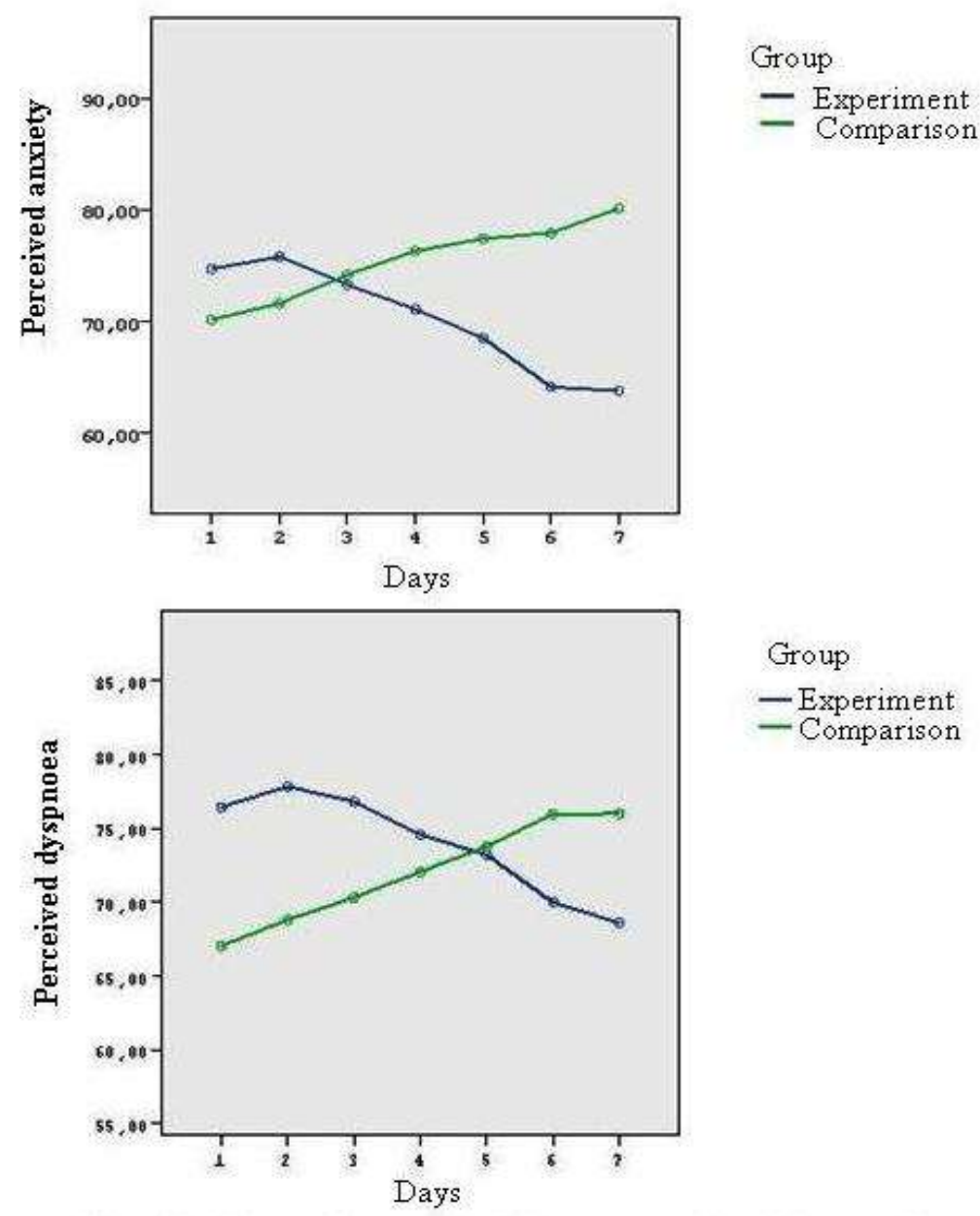

Figure 2. Changes in perceived dyspnoea and anxiety over time at baseline (day 1), and during (days 2-6) and follow-up (day 7 ).

The data are shown as mean.

Level of anxiety as measured by the VAS (0-100) at baseline was $74.71 \pm 7.41$ for the experimental group and $70.17 \pm 8.98$ for the control group, and indicated that patients using mechanical ventilation had high levels of perceived anxiety. There was a statistically significant difference between groups at baseline $(\mathrm{t}=2.63, \mathrm{p}=0.01)$. In the experimental group, anxiety level gradually decreased from day 3 to a lower level on day 7. Conversely, control group anxiety levels gradually increased over time (Figure 2). All these results support the effectiveness of hand massage and acupressure therapy in decreasing perceived anxiety.

\section{Discussion}

We found that levels of perceived dyspnoea and anxiety were quite high in our study participants. These results, along with those of similar studies, show that patients having mechanical ventilation support have highly stressful experiences, and the resulting anxiety and dyspnoea might interfere with weaning from mechanical ventilation (Rotondi et al. 2002, Tsay et al. 2005). For that 
reason, interventions to decrease perceptions of anxiety and dyspnoea become vital in this population.

Our data revealed that patients having mechanical ventilation support in the massage and acupressure therapy group experienced a statistically significant improvement in perceived dyspnoea and anxiety and in physiological indicators except for oxygen saturation as compared to those in the control group. This difference may be due to the effect of acupressure therapy on various acupoints, such as the Neiguan (PC6) and Hegu (LI4). Acupuncture therapy promotes the release of neurotransmitters and adrenocorticotropic hormones and is thought to contribute to relaxation and anxiety reduction, such that perception of breathlessness may be improved (Hsu 1996, NIH 1997). Also, massage which may provides comfort by decreasing tender in muscles is the opposite of stress response. Eliminating anxiety or providing comfort can be obtained through the decrease in muscle spasms (Fraser \& Ross 1993). It is thought that the effects of two applications strengthen one another. The state of relaxation was further supported by the improvement in the physiological indicators of HR, RRs and blood pressure values over time in this study.

Our data demonstrated that non-invasive massage and acupressure reduced the state of anxiety and dyspnoea among mechanically ventilated patients; Tsay et al. (2005) investigated the efficiency of acupressure therapy in patients requiring mechanical ventilation support. They determined a statistically significant healing in dyspnoea, anxiety, and physiological indicators of dyspnoea in patients who were applied acupressure with COPD in the support of mechanical ventilation. However, we think that this decrease in the values is not significant enough to relieve patient clinically, but there was a continuous decrease over time. Many acupressure studies conducted with community patients with COPD have shown that this treatment might improve dyspnoea and anxiety. However, in many studies sample groups are composed of patients with COPD who are not using mechanical ventilation and live at home, it is thought that this fact may have increased the effect of this method (Jobst et al. 1986, Maa et al. 1997, Wu et al. 2004). Studies demonstrate that massage could be used in treatments of illnesses such as anxiety, stress and pain through decreasing muscle tension (Fraser \& Ross 1993, Dunn et al. 1995, Ersser et al. 1998, Hmwe et al. 2015, Au et al. 2015, Dreyer et al. 2015). In studies conducted by that Kolcaba et al. (2004, 2006) found that hand massage increased the comfort of nursing home patients. There are differences in sampling and methods between Kolcaba et al. $(2004,2006)$ and this research. Our study adds empirical support for the effectiveness of hand massage and acupressure therapy in patients having mechanical ventilation support. 
The study was in two intensive respiratory care units affiliated with two hospitals, and the findings may therefore not be generalizable to broader COPD populations requiring mechanical ventilation support. Nevertheless, the study, a randomized controlled clinical trial, offers valid data to support the possible effectiveness of hand massage and acupressure therapy.

\section{Conclusion and recommendations}

In conclusion, our results suggest that hand massage and acupressure therapy might effectively relieve perceived dyspnoea and anxiety and reduce physiological indicators of HRs, RRs and blood pressure values in patients having mechanical ventilation support. Although the factors of patients having mechanical ventilation support and effects of many variables connected to the intensive care environment (such as catheters, intravenous infusion sets, monitors, mechanical tools, too much contact with the patient during the therapy and caring and etc.) might have affected negatively to the massage effectiveness, there was a statistically significant difference in the physiological indicators of anxiety and perceived anxiety and dyspnoea except for oxygen saturation.

The study provides a research-based intervention model for clinicians caring for patients having mechanical ventilation support. The application of these findings might have important implications for patients having mechanical ventilation support with high levels of perceived dyspnoea and anxiety. Assessment of dyspnoea and anxiety levels of these patients should be an essential part of nursing practice, and clinician nurses might want to consider providing hand massage and acupressure therapy as a method for improving symptoms of dyspnoea and anxiety. Nurses and patients' families could be easily trained to administer this therapy. The protocol for acupressure therapy is very simple, involving only two acupoints located on the hands. Also, hand massage and acupressure techniques can be easily learned and applied in clinical settings.

This study provides a foundation for future studies of massage and acupressure therapy in managing patients having mechanical ventilation support. Future researchers should replicate and expand upon the study to address basic research questions, and include a larger sample with longitudinal design in order to state with confidence that massage treatment and acupressure therapy are responsible for lower perceptions of dyspnoea and anxiety in patients having mechanical ventilation support. Subsequently, participants should be followed up to assess whether those who received hand massage and acupressure therapy stayed on the ventilator for a longer length than those having on treatment. This might add still more empirical data to support the effectiveness of hand massage and acupressure therapy. Finally, in studies of ICU patients who are 
Çınar Yücel, Ş., \& Eşer, İ. (2015). Effects of hand massage and acupressure therapy for mechanically ventilated patients. International Journal of Human Sciences, 12(2), 881-896. doi:10.14687/ijhs.v12i2.3054

suffering from dyspnoea and anxiety, difficulties may be encountered in gaining agreement to participate.

In accordance with the research results; using of different alternative methods except for massage and acupressure therapy, investigation massage and acupressure therapy separately, prolongation of the duration of hand massage and acupressure, and application in isolated intensive care units or house environment are all suggested for patients having mechanical ventilation support for the purpose of decreasing anxiety.

\section{Acknowledgements}

We would like to thank all the participants. We are also grateful to the physicians and nurses in the respiratory intensive care units for supporting the study.

\section{References}

Palm KH, Decker WW. (2003). Acute exacerbations of chronic obstructive pulmonary disease. Emergency Medicine Clinics of North America, 21: 331-352.

Thomas LA.(2003). Patient perceptions of the mechanical ventilation experience. Advanced Practice in Acute \& Critical Care, 14: 73-81.

Chlan LA (2009). Review of the Evidence for Music Intervention to Manage Anxiety in Critically Ill Patients Receiving Mechanical Ventilatory Support. Archives of Psychiatric Nursing, 23: 177179.

Johnson M, Sexton D. (1990). Distress during mechanical ventilation: patient's perceptions. Critical Care Nurse, 10: 48-57.

Kress J, Hall J. (2006). Sedation in the mechanically ventilated patient. Critical Care Medicine, 34: 2541-2546.

Rotondi AJ, Chelluri L, Sirio C, Mendelsohn A, Schulz R, Belle S, Im K, Donahoe M, Pinsky MR. (2002). Patients' recollections of stressful experience while receiving prolonged mechanical ventilation in an intensive care unit. Critical Care Medicine, 30: 746-752.

Tsay LS, Wang JC, Lin KC, Chung UL. (2005). Effects of acupressure therapy for patients having prolonged mechanical ventilation support. Journal of Advanced Nursing, 52: 142-150.

Korhan EA, Khorshid L, Uyar M. (2011). The effect of music therapy on physiological signs of anxiety in patients receiving mechanical ventilatory support. Journal of Clinical Nursing, 20: 1026-34.

Sarıcaoğlu F, Akıncı SB, Dal D, Aypar U. (2007). Yoğın Bakım Hastalarnda Analjęi ve Sedasyon. Turkey. Available from URL: http://medinfo.hacettepe.edu.tr/tebad/dergidoc/2005. Accessed 11 May 2007.

Fraser J, Ross K. (1993). Psycho-physiological effects of back massage on elderly institutionalized patients. Journal of Advanced Nursing, 18: 238-245.

Dunn C, Sleep J, Collett D. (1995). Sensing an improvement: an experimental study to evaluate the use of aromatherapy massage and periods of rest in an intensive care unit. Journal of Advanced Nursing, 21: 34-40.

Ersser SJ, Bentley T, Chappell S, Taylor R, Wade S, Walsh R, Bentley T. (1998). Sleep of Old People in Nursing Care Settings and the Impact of Back Massage on Sleep: a Pilot Study. Oxford Centre for Health Care Research \& Development Oxford Brookes University, Oxford. 
Kolcaba K, Schirm V, Steiner R. (2006). Effects of hand massage on comfort of nursing home residents. Geriatric Nursing, 27: 85-91.

Kolcaba K, Dowd T, Steiner R, Mitzel A. (2004). Efficacy of hand massage for enhancing the comfort of hospice patients. Journal of Hospice and Palliative Nursing, 6: 91-102.

National Institute of Health. Acupuncture. (2008). Available from URL: http://odp.od.nih.gov/consensus/cons/107/107 statement.htm. Accessed 12 December 2008.

Tsay SL, Chen ML. (2003). Acupressure and quality of sleep in patients with end-stage renal disease - a randomized controlled trial. International Journal of Nursing Studies, 40: 1-7.

Tsay SL. (2004). Acupressure and fatigue in patients with end-stage renal disease a randomized controlled trial. International Journal of Nursing Studies, 41: 99-106.

Hmwe NT, Subramanian P, Tan LP, Chong WK. (2015). The effects of acupressure on depression, anxiety and stress in patients with hemodialysis: a randomized controlled trial. International Lournal of Nursing Studies, 52(2):509-18.

Au DW, Tsang HW, Ling PP, Leung CH, Ip PK, Effects of acupressure on anxiety: a systematic review and meta-analysis. Acupuncture in Medicine, May 22. pii: acupmed-2014-010720. doi: 10.1136/acupmed-2014-010720.

Dreyer NE, Cutshall SM, Huebner M, Foss DM , Lovely JK, Bauer BA, Cima RR. (2015). Effect of massage therapy on pain, anxiety, relaxation, and tension after colorectal surgery: A randomized study. Complementary Therapies in Clinical Practice, 21: 154-159.

Hsu DT. (1996). Acupuncture: a review. Regional Anaesthesia, 21: 361-370.

Jobst K, Mcpherson K, Brown V, Fletcher HJ, Mole P, Chen JH, Arrowsmith J, Efthimiou J, Maciocia G, Shifrin K, Lane DJ. (1986). Controlled trial of acupuncture for disabling breathlessness. Lancet, 328: 1416-1419.

Maa SH, Gauthier D, Turner M. (1997). Acupressure as an adjunct to a pulmonary rehabilitation program. Journal of Cardiopulmonary Rehabilitation, 17: 268-276.

Wu HS, Wu SC, Lin JG, Lin LC. (2004). Effectiveness of acupressure in improving dyspnea in chronic obstructive pulmonary disease. Journal of Advanced Nursing, 45: 252-259.

Yang X, Liu X, Lou H. (1994). Clinical observation on needing extra channel points in treating mental depression. Journal of Traditional Chinese Medicine, 14: 14-18.

Eich H, Agelink MW, Lehmann E, Klieser E. (2000). Acupuncture in patients with minor depressive episodes and generalized anxiety. Fortschritte der Neurologie-Psychiatrie, 68: 137-144.

Wang SM, Kain ZN. (2001). Auricular acupuncture: a potential treatment for anxiety. Anaesthesia Analgesia, 92: 548-553. 